\title{
Millimetric radio continuum investigations of active galaxies with the BIMA array
}

\author{
Rogemar A. Riffel ${ }^{1}$, Dalia S. Reichert ${ }^{1}$ and Everton Lüdke ${ }^{1}$ \\ ${ }^{1}$ Universidade Federal de Santa Maria, CCNE - Departamento de Física - LARIE, Campus \\ Universitário, 97150-900 Santa Maria RS, Brazil
}

\begin{abstract}
In this paper we present some preliminary results of a 3-mm (86 GHz) radio continuum survey of selected Seyfert galaxies made with the Berkley-Maryland Illinois Millimeter Array (BIMA) in D configuration. The images shown here have spatial resolutions of about two arcminutes after gaussian tapering. At this observing wavelength, we expect to have a negligible non-thermal emission as the spectra is certainly dominated by free-free radio emission. We have measured flux densities and $3 \mathrm{~mm}$ luminosities which agree with a thermal blackbody spectra expected from data obtained with the IRAS satellite at wavelengths shorter than $80 \mu \mathrm{m}$. Data is presented for NGC3982, NGC5597, NGC5253, NGC1667, and NGC2997 and the main astrophysical consequences of such studies are discussed in this poster. We also argue that radio continuum at millimeter wavelengths is a better observational tool to study the star-formation in active galaxies than conventional infrared photometry.
\end{abstract}

\section{Introduction}

Recent molecular line observations of Seyfert galaxies include those from single-dish millimetre observations of CO and HCN lines at 3 and $1 \mathrm{~mm}$ wavelength (Curran 1998). In fact, Seyfert 2 galaxies show higher $L_{C O} / L_{B}$ ratios than Seyfert 1 galaxies, reaching about three times the values found for non-active galaxies (Heckman et al. 1989). In addition, millimetre line observations are useful to study the Seyfert phenomena, since molecular rings do exist in Seyfert galaxies (Myers \& Scoville 1987; Irwin \& Sofue 1998; Bergman et al. 1992; Miyoshi et al. 1995). Previous BIMA observations show the molecular gas, i.e. HCN and CO emission, concentrated around the active nucleus (Helfer \& Blitz 1997; Helfer \& Blitz 1995; Helfer \& Blitz 1993) and that the molecular ring is mostly aligned with the galactic disk seen in $\mathrm{H}_{\alpha}$ plates (Mcleod \& Rieke 1995; Maiolino \& Rieke 1995), which is particularly true for the powerful Seyfert 2 galaxy NGC4945 (Ott et al. 2001). The molecular gas content is also related to other important observational properties of Seyfert galaxies (Reichert et al. 2004).

In this work, we observed selected Seyfert galaxies and the archetypical starburst NGC5253 to look for relationships among the millimetre and infrared radio continuum properties, to look for alternatives to the standard infrared photometric method to probe star-formation in AGNs.

\section{The BIMA Observations}

The BIMA observations have been made with the shortest baseline configuration (Darray) with maximum baseline length of $8900 \mathrm{k} \lambda$. The on-source observing time was about 6 hours, carried out in a single track, on 25th July 1999.

The primary amplitude and bandpass calibrators were Mars and $3 \mathrm{C} 273$, the latter with a flux density of $22.5 \mathrm{Jy}$ at $3.5 \mathrm{~mm}$. 3C454.3 was also used as a control amplitude calibrator with a derived flux density of 7.2 Jy. An $830-\mathrm{MHz}$ wide continuum channel has 
also been recorded to produce the $3 \mathrm{~mm}$ image, which was strong enough to be used to self-calibrate de visibilities. The atmospheric absorption was strong for southern galaxies with $T_{\text {sys }}$ reaching $380 \mathrm{~K}$, but the data could be self-calibrated even in such conditions.

Data calibration (amplitude, phase and bandpass) were performed using standard procedures with the ATNF Miriad software. The calibrated data has been transported to the NRAO AIPS package for self-calibration and imaging. A uniform weighting scheme has been used with a gaussian taper of $400 k \lambda$, to produce the final images with a circular restoring beam of $200^{\prime \prime}$ so that they can be compared with available images obtained with the HST and various large optical telescope.

\section{Discussion and conclusions}

Our BIMA images and the IRAS spectral data for individual objects are available upon request. We see that the 3 -mm luminosities are lower, since the non-thermal contribution from the active nucleus and the interstellar matter vanishes at such short wavelength, and the thermal gas plus dust is actually detected. Hence, at millimetre radio window, we expect to have a negligible non-thermal emission as the spectra is certainly dominated by free-free radio emission with a contribution from the warm dust heated by stellar clusters.

A clear trend among the galaxies in which the 21-cm and far-infrared luminosities do correlate (Condon et al. 1991 see also Bohrer-Adornes \& Lüdke 2004 for recent ATCA observations of Seyfert galaxies). The data also suggests a positive correlation between $\mathrm{L}_{3 m m}$ and $\mathrm{L}_{I R}$ quantities. Therefore we present the preliminary result that the $\mathrm{L}_{3 m m}$ luminosity is a better indicator of the star formation than the available infrared data. Of course, further observations must be carried out to pursue better statistics.

Finally, we conclude that radio continuum imaging at millimeter wavelengths is a better observational tool to study the star-formation in active galaxies than conventional infrared photometry, since modern radioastronomical techniques provide spatial resolutions of about $100^{\prime \prime}$ or less, allowing to probe the inner $100 \mathrm{pc}$ field around the active nuclei in Seyfert galaxies.

\section{Acknowledgements}

The BIMA radio observatory is a consortium among University of Maryland, University of Illinois and UCLA on behalf of NSF, USA.

\section{References}

Bergman, P., Aalto, S., Black, J. H., \& Rydbeck, G. 1992, A\&A, 265, 403

Bohrer-Adornes, R., \& Lüdke, E. 2004, IAU Symposium No.222, these proceedings

Condon J. J., Anderson, M. L., \& Helou, G. 1991, ApJ, 376, 95

Curran, S. J. 2000, PhD thesis, Chalmers University of Technology, Sweden

Heckman, T. M., Blitz, L., Wilson, A. S., Armus, L., \& Miley, G. K. 1989, ApJ, 342, 735

Helfer, T. T., \& Blitz, L. 1997, ApJ, 478, 233

Helfer, T. T., \& Blitz, L. 1995, ApJ, 450, 90

Helfer, T. T., \& Blitz, L. 1993, ApJ, 419, 86

Irwin, J. A., \& Sofue, Y. 1992, ApJ, 396, L75

Maiolino, R., \& Rieke, G. H. 1995, ApJ, 454, 95

McLeod, K. K., \& Rieke, G. H. 1995, ApJ, 441, 96

Miyoshi, M., et al. 1995, Nature, 373, 127

Myers, S. T., \& Scoville, N. Z. 1987, ApJ, 312, L39

Ott, M., Whiteoak, J. B., Henkel, C., \& Wielebinski, R. 2001, A\&A, 368, 797

Reichert, D. S., Riffel, R. A., \& Lüdke, E. 2004 IAU Symposium No. 222, these proceedings 